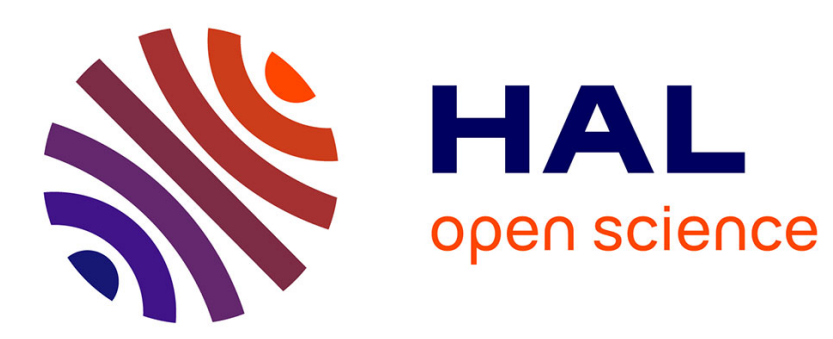

\title{
On extreme events for non-spatial and spatial branching Brownian motions
}

\author{
Jean Avan, Nicolas Grosjean, Thierry Huillet
}

\section{To cite this version:}

Jean Avan, Nicolas Grosjean, Thierry Huillet. On extreme events for non-spatial and spatial branching Brownian motions. Physica D: Nonlinear Phenomena, 2015, 298, pp.13-20. 10.1016/j.physd.2015.01.008 . hal-00950655v2

\section{HAL Id: hal-00950655 \\ https://hal.science/hal-00950655v2}

Submitted on 10 Feb 2015

HAL is a multi-disciplinary open access archive for the deposit and dissemination of scientific research documents, whether they are published or not. The documents may come from teaching and research institutions in France or abroad, or from public or private research centers.
L'archive ouverte pluridisciplinaire HAL, est destinée au dépôt et à la diffusion de documents scientifiques de niveau recherche, publiés ou non, émanant des établissements d'enseignement et de recherche français ou étrangers, des laboratoires publics ou privés. 


\title{
ON EXTREME EVENTS FOR NON-SPATIAL AND SPATIAL BRANCHING BROWNIAN MOTIONS
}

\author{
JEAN AVAN, NICOLAS GROSJEAN AND THIERRY HUILLET
}

\begin{abstract}
We study the impact of having a non-spatial branching mechanism with infinite variance on some parameters (height, width and first hitting time) of an underlying Bienaymé-Galton-Watson branching process. Aiming at providing a comparative study of the spread of an epidemics whose dynamics is given by the modulus of a branching Brownian motion (BBM) we then consider spatial branching processes in dimension $d$, not necessarily integer. The underlying branching mechanism is either a binary branching model or one presenting infinite variance. In particular we evaluate the chance $p(x)$ of being hit if the epidemics started away at distance $x$. We compute the large $x$ tail probabilities of this event, both when the branching mechanism is regular and when it exhibits very large fluctuations.
\end{abstract}

Keywords: Bienaymé-Galton-Watson process with infinite variance, height and width and hitting time, spatial branching Bessel process, evolutionary genetics and epidemics, extreme events.

\section{INTRODUCTION AND OUTLINE OF THE RESUltS}

The aim of this paper is a comparative study of the spread of an epidemics whose dynamics is given by the modulus of a branching Brownian motion (BBM) in dimension $d$, not necessarily integer; the underlying branching mechanism is either the one of a binary branching model or the one presenting infinite variance which we define presently.

Before the spatial aspects of the BBM are addressed, we study the impact of having a branching mechanism with infinite variance on the shape of the underlying continuous-time Bienaymé-Galton-Watson tree process. This chiefly concerns the time to extinction (the height of the tree), the maximum population size (the width of the tree) and its first hitting time. We compute the laws of these shape quantities for both the binary and the infinite variance branching mechanisms, in the sub-, super- and critical regimes, and we compare the two situations. The obtained results are developed in Section 2.

In Section 3, space is introduced. The special spatial BBM in dimension $d=1$ is addressed specifically since the model is then exactly solvable. Following the work of [20], we study the probability $p(x)$ that the Eve particle starting at 0 has some descendant ever diffusing above the threshold $x \geq 0$. The main new aspects of our results concerns the branching mechanism with infinite variance and its comparison 
with the binary branching model typically studied in [20] where the new individuals that come to birth along the branching mechanism are viewed as new mutants in an infinite allele model of population genetics.

In Section 4 , we deal with the $d \neq 1$ case. We study $p(x)$, the probability that the Eve particle starting at a distance $x$ of the origin has any of its descendants ever diffusing within a ball of radius $\epsilon$ centered at the origin. It is found that $p(x)$ satisfies a non-linear differential equation, which we use to compute its tail probabilities. In the critical case, the equation exhibits exact conformal covariance and has a corresponding invariant power-law solution. The large $x$ behavior of $p(x)$ is then in any case power-law, the exponent of which depends sharply on the value of the dimension $d$ with respect to a critical dimension $d_{c}$, reflecting the very large fluctuations of the branching mechanism. In the sub- and super-critical case, the large $x$ behavior of $p(x)$ is exponential with a multiplicative power prefactor depending on dimension $d$.

\section{BRANCHING PROCESSES: A REMINDER}

2.1. Generalities and well-known facts. Let us start with well-known facts on continuous-time elementary branching Bienaymé-Galton-Watson processes, [13].

Suppose at some random (mean one) exponential time, one initial individual dies out and produces a random number $M$ of offspring, with $M \in\{0,1,2, \ldots\}$. Let $f(z)=\mathbf{E}\left(z^{M}\right), z \in[0,1]$, be the probability generating function (pgf) of $M, f(z)=$ $\sum_{k \geq 0} \pi_{k} z^{k}$ with $\pi_{k}=\mathbf{P}(M=k)$.

Let $\phi_{t}(z)=\mathbf{E}\left(z^{N_{t}}\right), \phi_{0}(z)=z$, be the pgf of the number of particles $N_{t}$ alive at time $t \geq 0$. Then, setting $g(z)=f(z)-z, \phi_{t}(z)$ solves

$$
\dot{\phi}_{t}(z)=g\left(\phi_{t}(z)\right), \phi_{0}(z)=z
$$

where the '?' represents partial differentiation with respect to time.

We assume in the sequel that $\mu:=f^{\prime}(1)=\mathbf{E}(M)<\infty$.

If $\mu:=f^{\prime}(1)=\mathbf{E}(M)<1$, the process is denoted "subcritical". It is supercritical if $\mu>1$ and critical if $\mu=1$.

When the process is either critical or subcritical, extinction occurs with probability 1 , meaning $N_{\infty}=0$; otherwise if it is supercritical, extinction occurs with probability $\rho<1$ which is the smallest solution to $f(\rho)=\rho(g(\rho)=0)$. Note that $f^{\prime}(\rho)<1$. A supercritical process explodes $\left(N_{\infty}=\infty\right)$ with complementary probability $\bar{\rho}=1-\rho>0$

The probability that the time to extinction, say $\tau_{e}$, is smaller than $t$ is $\phi_{t}:=\phi_{t}(0)=$ $\mathbf{P}\left(N_{t}=0\right)=\mathbf{P}\left(\tau_{e} \leq t\right)$, solution to

$$
\dot{\phi}_{t}=g\left(\phi_{t}\right), \phi_{0}=0 \text {. }
$$

Alternatively, the probability that the time to extinction $\tau_{e}$ is larger than $t$ is $\bar{\phi}_{t}:=1-\phi_{t}(0)=\mathbf{P}\left(N_{t}>0\right)=\mathbf{P}\left(\tau_{e}>t\right)$, solution to

$$
\dot{\bar{\phi}}_{t}=-h\left(\bar{\phi}_{t}\right), \bar{\phi}_{0}=1 \text {, }
$$


where $h(z)=g(1-z) \cdot \tau_{e}$ is also called the height of the Bienaymé-Galton-Watson tree.

Whenever $M$ has all its moments finite, it holds that

$$
h(z)=(1-\mu) z+\sum_{k \geq 2} \mathbf{E}\left[(M)_{k}\right] \frac{(-z)^{k}}{k !},
$$

where $\mathbf{E}\left[(M)_{k}\right]:=\mathbf{E}[M(M-1) \ldots(M-k+1)]$ are the falling factorial moments of $M$.

We now specialize to two main special branching mechanisms generating BienayméGalton-Watson trees, one extremely classical, the other one not so much:

1. (binary branching) $f(z)=\pi_{0}+\pi_{1} z+\pi_{2} z^{2}$. Here $h(z)=(1-\mu) z+2 \pi_{2} \frac{z^{2}}{2 !}$, with $\mu=1-\left(\pi_{0}-\pi_{2}\right)$ and $\mu>1$ iff $\pi_{2}>\pi_{0}$.

2. (infinite variance Lamperti branching model [17]): $f(z)=1-\mu(1-z)+$ $C(1-z)^{\gamma}$ where $\mu / \gamma>C>\mu-1$ and $\gamma \in(1,2)$ so that $f(0) \in(0,1)$. Here $h(z)=(1-\mu) z+C z^{\gamma}$ and since $\gamma \in(1,2)$, the variance of $M$ is infinite, in contrast with the preceding binary splitting model. Given our constraints on $C$, $f(z)$ is a well-defined completely monotone pgf (in particular, $f^{\prime}(z)>0$, for all $z \in(0,1))$.

The probability system for this model is

(2) $\pi_{0}=1-\mu+C, \pi_{1}=\mu-C \gamma, \pi_{k}=C \frac{\gamma(\gamma-1)(2-\gamma) \ldots(k-\gamma-1)}{k !}, k \geq 2$.

Let us investigate $\bar{\phi}_{t}=\mathbf{P}\left(\tau_{e}>t\right)$ for these 2 models. We compute the exact form of the function and its asymptotic behavior at infinite time:

Consider first Model 1. This is easy to establish and very well-known, see [16] for instance:

Proposition 1. -a) In the subcritical case $\mu<1$ and $\bar{\rho}=0$ :

$\mathbf{P}\left(\tau_{e}>t\right)=e^{-(1-\mu) t} /\left(1+\pi_{2}\left(1-e^{-(1-\mu) t}\right) /(1-\mu)\right) \sim\left(1+\pi_{2} /(1-\mu)\right)^{-1} e^{-(1-\mu) t}$ with exponential tails.

-b) In the critical case $\mu=1$ and $\bar{\rho}=0$ :

$\mathbf{P}\left(\tau_{e}>t\right)=1 /\left(1+\pi_{2} t\right)$ with power-law Pareto(1) tails.

-c) In the supercritical case $\mu>1$ and $1>\bar{\rho}>0$ :

$\mathbf{P}\left(\tau_{e}>t\right)=e^{(\mu-1) t} /\left(1+\pi_{2}\left(e^{(\mu-1) t}-1\right) /(\mu-1)\right) \rightarrow(\mu-1) / \pi_{2}=\bar{\rho}$.

Proof: direct resolution.

Note $\mathbf{P}\left(\tau_{e}>t\right)=\bar{\rho}+O\left(e^{-(\mu-1) t}\right)$.

Consider now Model $\mathbf{2}$ which is not so widespread in the literature. We similarly establish: 
Proposition 2. -a) In the subcritical case $\mu<1$ and $\bar{\rho}=0$ : we get

$$
\begin{aligned}
\mathbf{P}\left(\tau_{e}>t\right) & =\left[e^{-(\gamma-1)(1-\mu) t} /\left(1+C\left(1-e^{-(\gamma-1)(1-\mu) t}\right) /(1-\mu)\right)\right]^{1 /(\gamma-1)} \\
& \sim(1+C /(1-\mu))^{-1 /(\gamma-1)} e^{-(1-\mu) t},
\end{aligned}
$$

again with exponential tails. The characteristic scale factor is $t_{c}=1 /(1-\mu)$, as in Model 1.

-b) In the critical case $\mu=1$ and $\bar{\rho}=0$ :

$\mathbf{P}\left(\tau_{e}>t\right)=(1+C(\gamma-1) t)^{-1 /(\gamma-1)}$ with power-law Pareto $(1 /(\gamma-1))$ tails. Here the tails of $\tau_{e}$ are lighter than in Model $\mathbf{1}$, due to $1 /(\gamma-1)>1$. Due to very large fluctuations of the offspring number, the time to extinction is stochastically shorter than the similar one under Model 1.

-c) In the supercritical case $\mu>1$ and $1>\bar{\rho}>0$ :

$$
\begin{aligned}
\mathbf{P}\left(\tau_{e}>t\right) & =\left[e^{(\gamma-1)(\mu-1) t} /\left(1+C\left(e^{(\gamma-1)(\mu-1) t}-1\right) /(\mu-1)\right)\right]^{1 /(\gamma-1)} \\
& \rightarrow \bar{\rho}=((\mu-1) / C)^{1 /(\gamma-1)},
\end{aligned}
$$

the probability of explosion. The characteristic scale factor is $t_{c}=1 /(1-\mu)$, as in Model 1.

Proof: again by direct computation.

Note that $\mathbf{P}\left(\tau_{e}>t\right)=\bar{\rho}+O\left(e^{-(\gamma-1)(\mu-1) t}\right)$. Given $\tau_{e}<\infty$, the tails of $\tau_{e}$ are exponential with a corrected scale factor $t_{c}=1 /[(\mu-1)(\gamma-1)]$. This fact is in contrast with what was observed in Model 1.

\subsection{Extreme events and the width of the Bienaymé-Galton-Watson tree.}

In this Section, we shall deal with extreme events pertaining to Bienaymé -GaltonWatson trees in continuous-time. To the best of our knowledge these issues have not yet been addressed in the literature. Although similar questions were raised and solved for discrete-time Bienaymé-Galton-Watson processes in [1], we shall see that in the very different continous-time context, the machinery involves ToeplitzHessenberg matrices which seems to be new.

We consider an extreme-event problem of interest: the maximal value (width of the Bienaymé-Galton-Watson tree) that $N_{t}$ can take in its lifetime. We need to expand the context of our study as follows: so far we have considered a single starting Eve particle. We now suppose there are $i$ initial particles, each branching independently of the others according to the same branching mechanism $f$. Then

$$
\phi_{t}(z)^{i}=\mathbf{E}\left(z^{N_{t}} \mid N_{0}=i\right)
$$

is the pgf of the whole population size $N_{t}$ at time $t$, given $N_{0}=i$.

Let us indeed define $p_{i}(k, t)=\mathbf{P}\left(N_{s} \leq k\right.$ for all $\left.s \leq t \mid N_{0}=i\right)$ as the probability that, starting from $i$ initial particles, $1 \leq i \leq k$, the population size profile keeps bounded above by $k$, up to time $t$. 
For all $i=1, \ldots, k$, from the Markov property ${ }^{1}$ we establish time evolution as:

Lemma 3. For all $i=1, \ldots, k$, with $p_{0}(k, t)=1$, we have

$$
\begin{aligned}
& \dot{p}_{1}(k, t)=-p_{1}(k, t)+\sum_{j=1}^{k} \pi_{j} p_{j}(k, t)+\pi_{0}, p_{1}(k, 0)=1, \\
& \dot{p}_{i}(k, t)=-i p_{i}(k, t)+i \sum_{j=i-1}^{k} \pi_{j-i+1} p_{j}(k, t), p_{i}(k, 0)=1, i=2, \ldots, k .
\end{aligned}
$$

Proof: We have:

$$
\begin{aligned}
p_{i}(k, t) & =e^{-i t}+i \int_{0}^{t} d s e^{-i s}\left(\sum_{j=0}^{k-i+1} \pi_{j} p_{i+j-1}(k, t-s)\right) \\
& =e^{-i t}+i \int_{0}^{t} d s e^{-i s} \sum_{j=i-1}^{k} \pi_{j-i+1} p_{j}(k, t-s) \\
& =e^{-i t}\left(1+i \int_{0}^{t} d \tau e^{i \tau} \sum_{j=i-1}^{k} \pi_{j-i+1} p_{j}(k, \tau)\right) .
\end{aligned}
$$

The term $e^{-i t}$ is the contribution corresponding to the first branching event occurring later than $t$ (the probability of which is the pdf of the minimum of $i$ iid exponential(1) random variables). The second term arises when the first branching event occurs at $s \leq t$, in which case, if the branching particle gives birth to $j$ particles, provided $i+j-1 \leq k, p_{i}(k, t)$ is given from $p_{i+j-1}(k, t-s)$ because the new starting number of particles is now $i+j-1$. Note that, if $i=1$, this equation exhibits the source term $p_{0}(k, \tau)=1$ occurring when $j=0$ with probability $\pi_{0}$.

We now rewrite this time evolution in vector form. Introduce

$$
\mathbf{p}(k, t):=\left(p_{i}(k, t), i=1, \ldots, k\right)^{\prime},
$$

and $Q_{k}$ the $k \times k$ upper Toeplitz-Hessenberg matrix with non-zero entries

$$
\begin{aligned}
Q_{k}(1,1)= & -\pi_{1}, Q_{k}(1, j)=-\pi_{j}, j=2, \ldots, k \text { and } \\
Q_{k}(i, i-1) & =-i \pi_{0}, Q_{k}(i, i)=i\left(1-\pi_{1}\right), \\
Q_{k}(i, j) & =-i \pi_{j-i+1}, i=2, \ldots, k ; j=i+1, \ldots, k,
\end{aligned}
$$

with $\mathbf{r}_{k}^{\prime}=\left(\pi_{0}, 0, \ldots, 0\right)^{2}$, we have the compact algebraic form $\left(\mathbf{1}^{\prime}=(1, \ldots, 1)\right.$ denotes the unit row vector)

$$
\dot{\mathbf{p}}(k, t)=-Q_{k} \mathbf{p}(k, t)+\mathbf{r}_{k}, \mathbf{p}(k, 0)=\mathbf{1} .
$$

Note that, with $\mathbf{k}^{\prime}:=(1,2, \ldots, k)$ and $D_{\mathbf{k}}=\operatorname{diag}(\mathbf{k}), Q_{k}=D_{\mathbf{k}} \bar{Q}_{k}$ for some $\bar{Q}_{k}$ involving only the $\pi_{j-i+1} \mathrm{~s}$ and $\bar{Q}_{k}=I-P_{k}$ for some substochastic matrix $P_{k}$

\footnotetext{
${ }^{1}$ The following results constitute the continuous-time version of similar results derived for discrete-time Bienayme-Galton-Watson processes in [1].

${ }^{2}$ Here and throughout all the paper, a bold $\mathbf{x}$ represents a column vector with appropriate dimension so that its transpose, say $\mathbf{x}^{\prime}$, is a row vector.
} 
with $P_{k} \mathbf{1}<\mathbf{1}$. From this structure of $Q_{k}$, this matrix is invertible with $Q_{k}^{-1}=$ $\left(I-P_{k}\right)^{-1} D_{\mathbf{k}}^{-1}$ and $\left(I-P_{k}\right)^{-1}$ is a potential matrix.

Lemma 4. The probability that, starting from $i$ initial particles, the overall maximum population size keeps bounded above by $k$ for ever, is given by: $p_{i}(k):=$ $\mathbf{e}_{i}^{\prime} Q_{k}^{-1} \mathbf{r}_{k}=\pi_{0} Q_{k}^{-1}(i, 1)$.

Remark: In particular, $p_{k}(k)=\pi_{0} Q_{k}^{-1}(k, 1)$ is the probability that, starting from $k$ initial particles, the overall maximum population size keeps equal to $k$ for ever.

Proof: The solution of the latter differential equation is

$$
\mathbf{p}(k, t)=e^{-Q_{(k)} t} \mathbf{1}+\int_{0}^{t} d s e_{k}^{-Q_{k} s} \mathbf{r}_{k}=e^{-Q_{k} t} \mathbf{1}+\left(I-e^{-Q_{k} t}\right) Q_{k}^{-1} \mathbf{r}_{k} .
$$

As $t \rightarrow \infty, p_{i}(k, t) \rightarrow \mathbf{e}_{i}^{\prime} Q_{k}^{-1} \mathbf{r}_{k}=\mathbf{P}\left(N_{t} \leq k\right.$ for all $\left.t \geq 0 \mid N_{0}=i\right)$ where $\mathbf{e}_{i}^{\prime}=$ $(0, \ldots, 0,1,0, \ldots, 0)$ is the $i$ th unit row vector of size $k$, with 1 in position $i$. Note that $p_{i}(k)-p_{i}(k-1)$ is the probability that, starting from $i$ initial particles, the overall maximum population size is exactly equal to $k$.

We now establish an interesting result on the joint probability and probability density of maximum size and its time-of-reach:

Lemma 5. Starting from $1 \leq i<k$ particles, the joint probability and probability density that the maximum population size is $k$ and that this maximum value is reached at time $t$ for the first time is given by:

$$
\left(p_{i}(k-1, t) \sum_{j=1}^{k-1} j e^{-t Q_{k-1}}(i, j) \pi_{k-j+1}\right) p_{k}(k) .
$$

\section{Proof:}

Let $1 \leq i<k$. The term $e^{-t Q_{k-1}}(i, j)$ is the probability, starting from $i$ particles, that $N_{t}=j<k$ given $N_{s} \leq k-1$ for all $s \leq t$. The term $p_{i}(k-1, t)$ is the probability that $N_{s} \leq k-1$ for all $s \leq t$, so the product of the two is the probability, starting from $i$ particles, that $N_{t}=j<k$ and $N_{s} \leq k-1$ for all $s \leq t$. Recall

$$
p_{i}(k-1, t)=\mathbf{e}_{i}^{\prime}\left(e^{-Q_{k-1} t} \mathbf{1}+\left(I-e^{-Q_{k-1} t}\right) Q_{k-1}^{-1} \mathbf{r}_{k-1}\right) .
$$

Now, if $t$ is a branching time for any of the $j$ particles alive at $t_{-}$,

$$
p_{i}(k-1, t) \sum_{j=1}^{k} j e^{-t Q_{k-1}}(i, j) \pi_{k-j+1}
$$

is the probability density that the first hitting time of $k$ is $t$ and that $k$ is the maximal value over the past. Multiplying this probability by $p_{k}(k)$, the probability that, starting from $k$ initial particles, the overall maximum population size stays lower or equal to $k$ for ever in the future, and making use of the independence of the past and the future gives the result.

Remarks: (i) Integrating (3) with respect to $t>0$, we obtain that

$$
p_{i}(k)-p_{i}(k-1)
$$


is the marginal probability that the maximum population size is $k \geq i$ given $N_{0}=i$. (ii) Summing (3) with respect to $k \geq i$ gives the marginal density of the first hitting time of the maximum over the lifetime.

A second result on size and (this time) overshot time is:

Lemma 6. Starting from $1 \leq i<k$ particles, the joint probability and probability density that the maximum population size over the past is $k$ and that this maximum value is overshot at time $t$ for the first time is:

$$
-\dot{p}_{i}(k, t) p_{k}(k)=\mathbf{e}_{i}^{\prime} e^{-Q_{k} t}\left(Q_{k} \mathbf{1}-\mathbf{r}_{k}\right) .
$$

\section{Proof:}

Let $1 \leq i<k$. Because with $\tau_{k}=\inf \left(s>0: N_{s} \geq k \mid N_{0}=i\right)$, defining the first overshooting time of $k,\left(N_{s} \leq k\right.$ for all $\left.s \leq t \mid N_{0}=i\right) \Rightarrow\left(\tau_{k}>t \mid N_{0}=i\right), p_{i}(k, t)$ is also $\mathbf{P}\left(N_{s} \leq k\right.$ for all $s \leq t$ and $\left.\tau_{k}>t \mid N_{0}=i\right)$. We now have

$$
\dot{p}_{i}(k, t)=\mathbf{e}_{i}^{\prime} e^{-Q_{k} t}\left(\mathbf{r}_{k}-Q_{k} \mathbf{1}\right)
$$

with

$$
\begin{aligned}
-\dot{p}_{i}(k, t) & =\mathbf{P}\left(N_{s}<k \text { for all } s<\tau_{k} \text { and } \tau_{k}=t \mid N_{0}=i\right) \\
& =\mathbf{e}_{i}^{\prime} e^{-Q_{k} t}\left(Q_{k} \mathbf{1}-\mathbf{r}_{k}\right),
\end{aligned}
$$

the joint probability that, given $N_{0}=i<k$, the maximum value of $N_{t}$ over the past is $k$ and that the first overshooting time density of this value $k$ occurs at $\tau_{k}=t$. Note that $Q_{k} \mathbf{1}-\mathbf{r}_{k}>\mathbf{0}$ as required from the substochasticity of $P_{k}$, if $-\dot{p}_{i}(k, t)$ is to be the probability density of some event.

We now explicitly compute the $Q_{k}^{-1}(i, 1)$, required in all previous lemmas in particular for

$$
p_{i}(k)=\mathbf{P}\left(N_{t} \leq k \text { for all } t \geq 0 \mid N_{0}=i\right)=\pi_{0} Q_{k}^{-1}(i, 1) .
$$

This can be achieved by introducing the generating function for $\theta$ coefficients as an ordinary power series:

$$
\theta(z):=\sum_{k \geq 1} \theta_{k} z^{k}
$$

with $\theta_{0}=0, \theta_{1}=\left(\left(1-\pi_{1}\right) / \pi_{0}\right), \theta_{k}=-\left(\pi_{k} / \pi_{0}\right), k \geq 2$. Of course we first have the trivial determinantal identity

$$
\left|Q_{k}\right|=k !\left|I-P_{k}\right| .
$$

From (2) in [14], we thus have the key expansion property expressing the generating function for the determinants as inverse of the original generating function:

$$
\frac{1}{1-\theta(z)}=\sum_{k \geq 0} \frac{\left|Q_{k}\right|}{k !}\left(z / \pi_{0}\right)^{k}=\sum_{k \geq 0}\left|I-P_{k}\right|\left(z / \pi_{0}\right)^{k} .
$$


Thus $\left|I-P_{k}\right|=\pi_{0}^{k}\left[z^{k}\right]\left(\frac{1}{1-\theta(z)}\right)$. By Faa di Bruno formula (see [7], p. 137) one then has:

$$
\left|I-P_{k}\right|=\pi_{0}^{k} B_{k}\left(\theta_{\bullet}\right)
$$

where

$$
B_{k}\left(\theta_{\bullet}\right)=\sum_{l=1}^{k} B_{k, l}\left(\theta_{\bullet}\right)
$$

are the complete Bell numbers of the sequence $\theta_{\bullet}=\left(\theta_{1}, \theta_{2}, \ldots\right)$, (see [7], p. 133), obtained by summing the ordinary Bell polynomials $B_{k, l}\left(\theta_{\bullet}\right)$ in the indeterminates $\theta_{\text {•. Now }}$

$$
Q_{k}^{-1}(i, 1)=\left(I-P_{k}\right)^{-1}(i, 1)=\frac{(-1)^{i+1}}{\left|I-P_{k}\right|} C_{1, i}
$$

where $C_{1, i}$ is the $(1, i)$-cofactor of $I-P_{k}$. Clearly now $C_{1, i}=\left(-\pi_{0}\right)^{i-1}\left|I-P_{k-i}\right|$. Using this, we finally obtain

Theorem 7. The probability

$$
p_{i}(k)=\pi_{0} Q_{k}^{-1}(i, 1)=\pi_{0} \frac{(-1)^{i+1}}{\left|I-P_{k}\right|} C_{1, i}=\left(\pi_{0}\right)^{i} \frac{\left|I-P_{k-i}\right|}{\left|I-P_{k}\right|}=\frac{B_{k-i}\left(\theta_{\bullet}\right)}{B_{k}\left(\theta_{\bullet}\right)},
$$

is a ratio of ordinary Bell numbers.

Corresponding expressions can be obtained for Model 2, while plugging in the $\pi_{k} \mathrm{~s}$, as given in (2).

As a simple illustration, let us finally compute the exact values and asymptotic behaviour of $p_{i}(k)$ for Model $\mathbf{1}$, using Theorem 7 and the fact that $\pi_{k}=0$ for $k \geq 3$.

Corollary 8. (i) In the subcritical case $\left(\pi_{0}>\pi_{2}\right)$, we have

$$
p_{i}(k)=\pi_{0} Q_{k}^{-1}(i, 1)=\left(\pi_{0}\right)^{i} \frac{\left|I-P_{k-i}\right|}{\left|I-P_{k}\right|}=\frac{1-\left(\frac{\pi_{2}}{\pi_{0}}\right)^{k-i+1}}{1-\left(\frac{\pi_{2}}{\pi_{0}}\right)^{k+1}} .
$$

When $k$ gets large, $p_{i}(k) \sim 1-\left(\frac{\pi_{2}}{\pi_{0}}\right)^{k-i+1} \rightarrow 1$ and $1-p_{i}(k)$ decreases geometrically with $k$. The term $1-p_{i}(k)$ is the probability that, starting from $i$ initial particles, the overall maximum population size overshoots $k$ at least once in the BienayméGalton-Watson process lifetime (before $\tau_{e}$ ).

(ii) The critical case $\left(\pi_{0}=\pi_{2}\right)$. Setting $\pi_{2}=\pi_{0}+\varepsilon$ in the latter formula, we get

$$
p_{i}(k) \underset{\varepsilon \rightarrow 0}{\sim} \frac{1-\left(1-\frac{\varepsilon}{\pi_{2}}(k-i+1)\right)}{1-\left(1-\frac{\varepsilon}{\pi_{2}}(k+1)\right)} \underset{\varepsilon \rightarrow 0}{\sim} 1-\frac{i}{k+1} .
$$

We conclude that $1-p_{i}(k)$ decreases algebraically like $i / k$ with $k$, hence much slower than in the subcritical case.

(iii) In the supercritical case $\left(\pi_{0}<\pi_{2}\right)$, if the process explodes, $p_{i}(k)=0$ and conditioned on non-explosion, we are taken back to the previous subcritical study with the new branching mechanism $f_{\rho}(z)=\rho^{-1} f(\rho z)$ where $\rho<1$ is the 
extinction probability solving $f(\rho)=\rho$, here $\rho=\pi_{0} / \pi_{2}$. Thus $f_{\rho}(z)=\rho^{-1} f(\rho z)=$ $\pi_{2}+\pi_{1} z+\pi_{0} z^{2}$, exchanging the roles of $\pi_{0}$ and $\pi_{2}$.

Proof: Only part $(i)$ requires a proof. With $z_{-}=\pi_{0} / \pi_{2}$, we have

$$
\frac{1}{1-\theta(z)}=\frac{1}{(1-z)\left(1-z / z_{-}\right)}=\frac{A}{1-z}+\frac{B}{1-z / z_{-}}
$$

where $A=-z_{-} /\left(1-z_{-}\right)$and $B=1 /\left(1-z_{-}\right)$. Thus

$$
\left|I-P_{k}\right|=\pi_{0}^{k} \frac{\pi_{0}}{\pi_{0}-\pi_{2}}\left(1-\left(\frac{\pi_{2}}{\pi_{0}}\right)^{k+1}\right) .
$$

Note that in this binary case, $B_{k}\left(\theta_{\bullet}\right)=\frac{\pi_{0}}{\pi_{2}-\pi_{0}}\left[1-\left(\frac{\pi_{2}}{\pi_{0}}\right)^{k+1}\right]$, in accordance with Theorem 7 .

\section{Spatial BRANChing PROCESS IN DIMENSION 1}

We have until now dealt with zero-space dimension tree-like branching processes, underlining how some basic shape parameters of the tree (either sub-, super- or critical) were strongly affected by the infinite-variance assumption.

We now move to the problem of the spatial Bienaymé-Galton-Watson process, first of all restricted to the one-dimensional case. This problem is in essence distinct and its significant features can therefore not be deduced by any obvious generalization from the zero-dimensional case. We shall revisit some results of [20] and extend them to a new situation akin to Model 2. In such a spatial branching process, an Eve particle diffuses according to one-dimensional standard Brownian motion (with diffusion constant fixed to 1 without loss of generality). At some (mean one) exponential time, it dies out giving birth in the process to $M$ offspring; if $M>0$, the daughter particles diffuse according to independent standard Brownian motions, started where the mother particle died. The study of branching Brownian motion (measure-valued processes) has a long and rich history, starting from [4], through [8], to [2], to cite only just a few. See also [10] and [19].

Let $p(x)$ be the probability that the Eve particle starting at 0 has some descendant ever diffusing above the threshold $x \geq 0$. Then $[20] p(x)$ solves $(p(0)=1)$

$$
\frac{1}{2} p^{\prime \prime}-h(p)=0 \text { or } p^{\prime}(x)^{2}-4 \int^{p(x)} h(z) d z=C t e,
$$

as a stationary solution of the Kolmogorov-Petrovsky-Piskounov equation, [15].

We note that $p(x)$ is also the probability that the supremum of the positions of all particles that appeared at any time exceeds $x$, so $p(x)=1-q(x)$ where $q(x)$ is a probability distribution function (which in particular is monotone non-decreasing).

Because $p(x) \underset{x \rightarrow \infty}{\rightarrow} \bar{\rho}$, the limit $p^{\prime}(x)$ should also exist and this limit is necessarily 0 . These equations are then generically solved by inverting the quadrature:

$$
x=\frac{1}{2} \int_{p(x)}^{1} H(y)^{-1 / 2} d y,
$$


where $H(y)=\int_{\bar{\rho}}^{y} h(z) d z$ (differentiating the latter equation with respect to $x$ and squaring the result gives back (5)). Let us investigate $p(x)$ for the previous two examples:

Consider first Model $\mathbf{1}$.

Proposition 9. - a) In the subcritical case $\mu<1$ and $\bar{\rho}=0$ :

$H(y)=\frac{(1-\mu)}{2} y^{2}\left(1+\frac{2 \pi_{2} y}{3(1-\mu)}\right)$. Then

$$
p(x)=\frac{4 A E(x)}{c(1-A E(x))^{2}}
$$

where $E(x)=\exp (-\sqrt{2(1-\mu)} x), c=\frac{2 \pi_{2}}{3(1-\mu)}$ and $A=(\sqrt{1+c}-1)^{2} / c$.

Note that $p(x)$ has exponential tails with scale factor $x_{c}=1 / \sqrt{2(1-\mu)}$ and $p(0)=1$.

-b) In the critical case $\mu=1$ and $\bar{\rho}=0$ : Here $p(x)=\left(1+x / x_{c}\right)^{-2}$ hence $p(x)$ decays algebraically at infinity with exponent 2 . The scale factor is $x_{c}=\sqrt{3 / \pi_{2}} / 4$.

-c) In the supercritical case $\mu>1$ and $1>\bar{\rho}>0$ : Here, with $\bar{\rho}=1-\pi_{0} / \pi_{2}$, $p(x)=\bar{\rho}+O\left(e^{-x / x_{c}}\right)$, where $x_{c}=1 / \sqrt{2 h^{\prime}(\bar{\rho})}$.

\section{Proof:}

-a) and -b) follow by direct computations. Concerning -c), $p(x)$ has an atom at infinity which is the probability of explosion of the underlying branching process and the remaining tails are exponential. Indeed, letting $p(x)-\bar{\rho}=\widetilde{p}(x), \widetilde{p}(x)$ solves $\frac{1}{2} \widetilde{p}^{\prime \prime}-h(\widetilde{p}+\bar{\rho})=0$ which for small $\widetilde{p}($ large $x)$ is $\frac{1}{2} \widetilde{p}^{\prime \prime}-h^{\prime}(\bar{\rho}) \widetilde{p}=0$, recalling $h(\bar{\rho})=0$. One can check that $h^{\prime}(\bar{\rho})=\pi_{2}-\pi_{0}>0$ and so $\widetilde{p}$ is exponential with the right scale factor.

As observed in [20], any branching model for which $f(z)=\pi_{0}+\pi_{1} z+\pi_{2} z^{2}+O\left(z^{\gamma^{\prime}}\right)$ with $\gamma^{\prime}>2$, will display similar tail behaviors.

Let us now switch to Model 2. We get:

Proposition 10. - a) In the subcritical case $\mu<1$ and $\bar{\rho}=0$ :

$H(y)=\frac{(1-\mu)}{2} y^{2}\left(1+\frac{2 C y^{\gamma-1}}{(\gamma+1)(1-\mu)}\right)$. One computes:

$$
p(x)=\left(\frac{4 A E(x)}{c(1-A E(x))^{2}}\right)^{1 /(\gamma-1)}
$$

where: 
$E(x)=\exp (-(\gamma-1) \sqrt{2(1-\mu)} x), c=\frac{2 C}{(\gamma+1)(1-\mu)}$ and $A=(\sqrt{1+c}-1)^{2} / c$; $p(x)$ has again exponential tails with scale factor $x_{c}=1 / \sqrt{2(1-\mu)}$.

-b) In the critical case $\mu=1$ and $\bar{\rho}=0$ : We get $p(x)=\left(1+x / x_{c}\right)^{-2 /(\gamma-1)}$ and $p(x)$ decays algebraically at infinity with exponent $2 /(\gamma-1)$. The scale factor is $x_{c}=\sqrt{(\gamma+1) / C} /(\gamma-1)$.

-c) In the supercritical case $\mu>1$ and $1>\bar{\rho}>0$ : Setting $\bar{\rho}=((\mu-1) / C)^{1 /(\gamma-1)}$, one has $p(x)=\bar{\rho}+O\left(e^{-x / x_{c}}\right)$, where $x_{c}=1 / \sqrt{2 h^{\prime}(\bar{\rho})} . \quad p(x)$ has an atom at infinity which is the probability of explosion (non-extinction) and the remaining tails are exponential.

\section{Proof:}

Statements -a) and -b) are obtained by direct computations. To get statement -c), setting as before $p(x)-\bar{\rho}=\widetilde{p}(x), \widetilde{p}(x)$ solves $\frac{1}{2} \widetilde{p}^{\prime \prime}-h(\widetilde{p}+\bar{\rho})=0$ which for small $\tilde{p}$ (large $x$ ) is $\frac{1}{2} \widetilde{p}^{\prime \prime}-h^{\prime}(\bar{\rho}) \tilde{p}=0$, recalling $h(\bar{\rho})=0$. One checks that here $h^{\prime}(\bar{\rho})=(\mu-1)(\gamma-1)>0$ and $\tilde{p}$ is exponential with the claimed scale factor, different from the scale factor obtained in the subcritical case.

The quantity $p(x)$ is equivalently the probability that an Eve particle started at $x \geq 0$ has some descendant ever diffusing below the threshold $x=0$. This way of thinking $p(x)$ also pertains to dimensions not equal to one which we move to now.

\section{Spatial BRAnChing process in Dimension $d \neq 1$}

Let $P(\mathbf{x})$ be the probability that some particle started at $\mathbf{x}$ in $\mathbb{R}^{d}(d=2,3, \ldots)$ has some descendant ever diffusing within a ball of radius $\varepsilon>0$ around the origin, with $x:=\|\mathbf{x}\|_{2}=\left(\sum_{i=1}^{d} x_{i}^{2}\right)^{1 / 2}>\varepsilon$. Then, from [20], introducing $\Delta$ as the $d$-dimensional Laplacian, $P(\mathbf{x})$ solves $\frac{1}{2} \Delta P-h(P)=0$ and in view of rotational invariance, $p(x):=P\left(\|\mathbf{x}\|_{2}\right)$ solves

$$
\frac{1}{2} p^{\prime \prime}+\frac{d-1}{2 x} p^{\prime}-h(p)=0,
$$

We impose the boundary conditions $p(\varepsilon)=1$ and $p(\infty)=0$ for consistency with the probabilistic interpretation of $p$. Indeed this modified construction is dictated by the fact that $d$-dimensional branching Brownian motion with $d=2,3$, .. has zero probability to meet the origin. $\frac{1}{2} \partial_{x}^{2}+\frac{d-1}{2 x} \partial_{x}$ is the Bessel generator of the modulus of a $d$-dimensional Brownian motion. Thus $p(x)$ is the probability that the full trail of the $d$-dimensional branching Brownian motion ever happened to be at distance to the origin less than $\varepsilon$. Hence the boundary conditions.

This construction can be extended to non-integer $d(\geq 2)$ as follows. Let $R_{t}=$ $\exp \left(B_{t}+a t\right)$ and $X_{t}=R_{\tau_{t}}$ where $\tau_{t}=\int_{0}^{t} X_{s}^{-2} d s$ and $B_{t}$ is the standard Brownian motion. Assume $a \geq 0$. Then the infinitesimal generator of $X_{t}>0$, as a timechanged geometric Brownian motion $R$ with non-negative drift, is [12]

$$
\frac{1}{2} \partial_{x}^{2}+\frac{(2 a+1)}{2 x} \partial_{x}
$$


so it is the generator of some Bessel process (say $\mathrm{BS}_{d}$ ), with 'dimension' parameter $d=2(a+1) \geq 2$, not necessarily an integer.

Proposition 11. Denote by $p(x)$ the probability that some branching $1-$ dimensional $B S_{d}$ particle system, started at $x>0$, has some descendant ever diffusing below $\varepsilon$ $(x>\varepsilon>0)$. Then $p(x)$ solves

$$
\frac{1}{2} p^{\prime \prime}+\frac{d-1}{2 x} p^{\prime}-h(p)=0,
$$

with $p(\varepsilon)=1$ and $p(\infty)=0$.

The $\mathrm{BS}_{d}$ process $X$ is well-defined even if $d>1$ then with $\mathbf{E}\left(\int_{0}^{t} d s / X_{s}\right)<\infty$, and also even if $d>0$, [11]. We also recall some basic properties of $\mathrm{BS}_{d}$ processes with respect to their dimension $d$ as from [18]:

- For $d>2$, the process $\mathrm{BS}_{d}$ is transient.

- For $d \geq 2$ the point 0 is polar and for $d \leq 1$ it is reached almost surely.

- For $0<d<2, \mathrm{BS}_{d}$ is (null) recurrent; the point 0 is instantaneously reflecting.

We are now in a position to extend the interpretation of the latter differential equation describing the $\mathrm{BS}_{d}$ process $X$ when $d$ is non integer. Integer values of $d$ all correspond to a $d$-dimensional Brownian motion with full rotational invariance and the occurrence of $d$ in the differential equation follows from the reduction of a $d$-dimensional Laplacian to invariant configurations. We consistently conjecture that non-integer values of $d$ similarly characterize Brownian motion on a fractal-type background (possibly relevant in epidemics propagation description) again with a full "rotational" invariance, here by the simplest analytic continuation of the differential equation to non-integer values of $d$ (More complicated analytic extensions involving additional, real-periodic functions may be considered but they shall not be addressed here). This bears some technical resemblance with procedures in quantum field theories such as dimensional regularization. This conjecture is strongly borne out by the following checks: starting from the definition of spherically symmetric random walks in non-integer dimensions $d$ by Bender et al. [6] and taking the large (continuous) limit of radii of the nested $d$-dimensional spheres between which the particle random-walks, one recovers exactly the drift contribution $\frac{d-1}{2 x}$ and the constant unit local variance term in the second-order differential operator generating $B S_{d}$. Following this interpretation, the $B S_{d}$ process $X_{t}$ may be viewed as the modulus of some isotropic $d$-dimensional diffusion process, evolving in a $d$-dimensional space for which the surface of a ball with radius $x$ is $2 \pi^{d / 2} x^{d-1} / \Gamma(d / 2)$.

We shall now study equation (6) when $h(z)=(1-\mu) z+C z^{\gamma}, \gamma \in(1,2)$. It is not solvable contrary to the $d=1$ case, except for integer values of $\gamma$ (elliptic functions for $\gamma=3$, hyperelliptic functions for $\gamma=4,5 \cdots$. These integer values however lie beyond the interval of relevance for the probabilistic interpretation of the model. It must be however suggested that rational values of $\gamma$ lying in the relevant open interval $(1,2)$ may still lead to solutions with some interpretation in algebraic geometry (multiple coverings of elliptic or Prym manifolds). 
Anyway here we limit ourselves to the sole asymptotic analysis (large $x$ ) form of the solutions with the suitable limit behavior $p(\infty)=0$ (except in one case).

The case $\gamma=2$ (Model $\mathbf{1}$ ) has been analyzed to a large extent (in the critical regime) by [20]. Some further extensions of the binary branching model (either subcritical or critical) has also been reported in [9], in the $d=2$ dimensional case, involving a deep study of the dynamics of both perimeter and area of the convex hull of the BBM trail.

4.1. Subcritical case $(\mu<1)$. Recalling again the asymptotic limit behavior $p(\infty)=0$ we conclude that the higher power term $C z^{\gamma}$ is to be dropped when analyzing around $\infty$. The large $x$ (small $p$ ) solutions are thus governed by

$$
\frac{1}{2} p^{\prime \prime}+\frac{d-1}{2 x} p^{\prime}-(1-\mu) p=0
$$

which can be mapped into a modified Bessel equation, (see [3], p. 117). Indeed, with $\alpha, \beta$ some constants, let

$$
p(x)=x^{\alpha} J_{\alpha}(\beta x),
$$

where $J_{\alpha}(x)$ obeys $J_{\alpha}^{\prime \prime}+J_{\alpha}^{\prime} / x+\left(1-\alpha^{2} / x^{2}\right) J_{\alpha}=0$, as a Bessel function of the first kind, of order $\alpha$. Then $p$ obeys

$$
p^{\prime \prime}-\frac{2 \alpha-1}{x} p^{\prime}+\beta^{2} p=0 .
$$

Setting $\alpha=(2-d) / 2$ and $\beta=i \sqrt{2(1-\mu)}=: i \gamma$ and recalling that $I_{\alpha}(x)=$ $i^{-\alpha} J_{\alpha}(i x)$ is the modified Bessel function of the first kind of order $\alpha$, we get:

$$
p(x)=x^{\alpha}\left(A_{1} I_{\alpha}(\gamma x)+A_{2} K_{\alpha}(\gamma x)\right),
$$

where $K_{\alpha}(x)$ is the modified Bessel function of the second kind of order $\alpha$.

Recalling $K_{\alpha}(x) \sim e^{-x} \sqrt{\pi /(2 x)}$ near $x=\infty$ and keeping only the decaying factor at $\infty$, we establish:

Proposition 12. In the subcritical case, $p(x)$ behaves for large $x$ as

$$
p(x) \sim A_{2} x^{\alpha} K_{\alpha}(\gamma x) \sim \lambda x^{-(d-1) / 2} e^{-\sqrt{2(1-\mu)} x}, \lambda>0
$$

Compared to the exact $d=1$ case studied before, the asymptotics of $p(x)$ exhibit an extra $x^{-(d-1) / 2}$ power term.

4.2. Critical case $(\mu=1)$. In this case, $h(p)=C p^{\gamma}$. A conformal covariance property then arises:

Proposition 13. If $p$ is a solution of (6) with $h(p)=C p^{\gamma}$, then, for all $\lambda>0$, $p_{\lambda}(x)=\lambda^{2 /(\gamma-1)} p(\lambda x)$ are also solutions. The constant $2 /(\gamma-1)$ is the conformal weight for $p^{3}$.

This will play an important role in the next analysis. In particular the conformal invariant solution $m(x)=x^{-2 /(\gamma-1)}$, will appear.

\footnotetext{
${ }^{3}$ By analogy with the terminology of Conformal Field Theory, the constant $\Delta:=2 /(\gamma-1)$ such that $p(x)$ is transformed into $\lambda^{\Delta} p(\lambda x), \lambda>0$, can be named a conformal weight, see [5].
} 
4.2.1. Behavior of $p$ near infinity. We first assume an asymptotic power-law form $p \sim \lambda x^{-\alpha}, \alpha, \lambda>0$, leading to

$$
\lambda \alpha x^{-(\alpha+2)}(\alpha+2-d)-2 C \lambda^{\gamma} x^{-\gamma \alpha}=0 .
$$

Let us first analyze the power-law behaviour. We need to impose $\alpha+2 \leq \gamma \alpha$, otherwise the dominant term would be the unique one $x^{-\gamma \alpha}$, which would be inconsistent. So $\alpha \geq 2 /(\gamma-1)$.

- Suppose first $\alpha>2 /(\gamma-1)$. Then necessarily the power-law exponent is $\alpha=d-2$ and this regime occurs when $d>d_{c}:=2+2 /(\gamma-1)$. Note that there is no specification of what $\lambda$ is (except of course for $\lambda>0$ ).

- Suppose now $\alpha=2 /(\gamma-1)$. Then the two power terms contribute equally likely and the solution asymptotically behaves like the conformally invariant monomial $m(x)=x^{-2 /(\gamma-1)}$, obeying $m(x)=\lambda^{2 /(\gamma-1)} m(\lambda x)$.

We can now discuss the scale factor $\lambda$.

* Suppose first $d \neq \alpha+2$; then we also need to have $d<d_{c}=2+\alpha=2+2 /(\gamma-1)$ in addition with

$$
\lambda \alpha+(\alpha+2-d)=2 C \lambda^{\gamma}
$$

leading to $\lambda=(\alpha(\alpha+2-d) / C)^{1 /(\gamma-1)}=\left(\left(d_{c}-d\right) /((\gamma-1) C)\right)^{1 /(\gamma-1)}$.

* Suppose now $d=\alpha+2$; then $d=d_{c}$ and we have to try the enhanced asymptotic form $p \sim \lambda x^{-\alpha}(\log x)^{\beta}, \alpha=d_{c}-2, \lambda>0$. We get

$$
\begin{aligned}
\frac{d-1}{x} p^{\prime} & \sim \lambda\left(d_{c}-1\right) x^{-(\alpha+2)}\left(\beta(\log x)^{\beta-1}-\alpha(\log x)^{\beta}\right) \\
p^{\prime \prime} & \sim \lambda x^{-(\alpha+2)}\left(\alpha(\alpha+1)(\log x)^{\beta}-\beta(2 \alpha+1)(\log x)^{\beta-1}+\beta(\beta-1)(\log x)^{\beta-2}\right) .
\end{aligned}
$$

Plugging these estimates into $p^{\prime \prime}+\frac{d-1}{x} p^{\prime}-2 h(p)=0$, the $(\log x)^{\beta}$ terms cancel, leading to

$$
-\alpha \lambda \beta x^{-(\alpha+2)}(\log x)^{\beta-1}-2 C \lambda^{\gamma}(\log x)^{\beta \gamma} x^{-\alpha \gamma}=0,
$$

discarding the $(\log x)^{\beta-2}$ term as compared to $(\log x)^{\beta-1}$. Observing $\alpha \gamma=\alpha+2$, this can be achieved only if $\beta \gamma=\beta-1$, so if $\beta=-1 /(\gamma-1)$. The constant $\lambda$ is also determined by $\lambda^{\gamma-1}=-\alpha \beta /(2 C)$, so $\lambda=\left(C(\gamma-1)^{2}\right)^{-1 /(\gamma-1)}$.

To summarize, we have shown:

Theorem 14. In the critical case, the behavior of $p(x)$ for large $x$ depends on the value of the dimension $d$ with respect to a critical dimension $d_{c}:=2+2 /(\gamma-1)$.

- if $d>d_{c}, p \sim \lambda x^{-(d-2)}$ with $\lambda>0$ being left unspecified.

- if $d=d_{c}, p \sim \lambda\left(x^{-2} / \log (x)\right)^{\left(d_{c}-2\right) / 2}$ with $\lambda=\left(C(\gamma-1)^{2}\right)^{-1 /(\gamma-1)}$.

- if $d<d_{c}, p \sim \lambda x^{-2 /(\gamma-1)}$ with $\lambda=\left(\left(d_{c}-d\right) /((\gamma-1) C)\right)^{1 /(\gamma-1)}$. 
4.2.2. Behavior of $p$ near the origin. Although this question does not necessarily make sense in our probabilistic context because $p(x)$ is intrinsically defined for $x>\varepsilon$, it turns out that the formal analysis of $p(x)$ near the origin is possible.

We first try the asymptotic power-law form $p \sim \lambda x^{-\beta}, \beta, \lambda>0$, leading to

$$
\lambda \beta x^{-(\beta+2)}(\beta+2-d)-2 C \lambda^{\gamma} x^{-\gamma \beta}=0 .
$$

Once again we first analyze the power-law behaviour. We need to impose $\beta+2 \geq \gamma \beta$, otherwise the dominant term would be the unique one $x^{-\gamma \beta}$, which would fail. So $\beta \leq 2 /(\gamma-1)$.

- Suppose first $\beta<2 /(\gamma-1)$. Then necessarily the power-law exponent is $\beta=d-2$. This regime occurs when $2<d<d_{c}:=2+2 /(\gamma-1)$. Note that there is again no specification of what $\lambda$ is (except of course for $\lambda>0$ ).

- Suppose now $\beta=2 /(\gamma-1)$. Then the two power terms contribute equally likely and we are back to the conformally invariant solution.

Let us now discuss the scale factor $\lambda$.

* Suppose first $d \neq \beta+2$; then we also need to have $d<d_{c}=2+\beta=2+2 /(\gamma-1)$ in addition with

$$
\lambda \beta+(\beta+2-d)=2 C \lambda^{\gamma},
$$

leading to $\lambda=(\beta(\beta+2-d) / C)^{1 /(\gamma-1)}=\left(\left(d_{c}-d\right) /((\gamma-1) C)\right)^{1 /(\gamma-1)}$.

* Suppose now $d=\beta+2$; then $d=d_{c}$ and we try the asymptotic form $p \sim$ $\lambda x^{-\beta}(-\log x)^{\delta}, \beta=d_{c}-2, \lambda>0$. We get

$$
\begin{aligned}
\frac{d-1}{x} p^{\prime} & \sim \lambda\left(d_{c}-1\right) x^{-(\beta+2)}\left(-\delta(-\log x)^{\delta-1}-\beta(-\log x)^{\delta}\right) \\
p^{\prime \prime} & \sim \lambda x^{-(\beta+2)}\left(\beta(\beta+1)(-\log x)^{\delta}+\delta(2 \beta+1)(-\log x)^{\delta-1}+\delta(\delta-1)(-\log x)^{\delta-2}\right) .
\end{aligned}
$$

Plugging these estimates into $p^{\prime \prime}+\frac{d-1}{x} p^{\prime}-2 h(p)=0$, the $(-\log x)^{\beta}$ terms cancel again, leading to

$$
\lambda \beta \delta x^{-(\beta+2)}(-\log x)^{\delta-1}-2 C \lambda^{\gamma}(-\log x)^{\delta \gamma} x^{-\beta \gamma}=0,
$$

discarding the $(-\log x)^{\delta-2}$ term compared to $(-\log x)^{\delta-1}$ when $x$ is small. Observing $\beta \gamma=\beta+2$, this could be achieved only if $\delta \gamma=\delta-1$, so if $\delta=-1 /(\gamma-1)$. The constant $\lambda$ should also be determined by $\lambda^{\gamma-1}=\beta \gamma /(2 C)$ and because $\beta<0$, $\lambda$ cannot be real ${ }^{4}$.

Interestingly enough we may also define consistent solutions of the alternative asymptotic form $p \sim c\left(1+\lambda x^{\beta}\right)$ for some constants $c, \beta>0$. To leading order, we need to have

$$
\lambda c \beta(\beta+d-2) x^{\beta-2}=2 c^{\gamma}
$$

\footnotetext{
${ }^{4}$ There exist solutions with a complex prefactor which we disregard, due to their lack of physical meaning so far, in particular because it hampers an interpretation of $p$ as a probability.
} 
which also requires $\beta=2$ together with $\lambda=c^{\gamma-1} / d>0$.

To summarize:

Proposition 15. In the critical case, the behavior of $p(x)$ near $x=0$ also depends on the value of the dimension $d$ with respect to the critical dimension $d_{c}$ (with $\left.d_{c}=2+2 /(\gamma-1)\right)$

- if $2<d<d_{c}, p \sim \lambda x^{-(d-2)}$ with $\lambda>0$ being left unspecified.

- if $0<d<d_{c}, p \sim \lambda x^{-2 /(\gamma-1)}$ with $\lambda=\left(\left(d_{c}-d\right) /((\gamma-1) C)\right)^{1 /(\gamma-1)}$.

- if $d>0, p \sim c\left(1+\lambda x^{2}\right)$ with $c>0$ and $\lambda=c^{\gamma-1} / d$.

- if $d=d_{c}$, there is no real solution of the form $\lambda x^{-\beta}(-\log x)^{\delta}$ with $\beta, \delta, \lambda$ real. A solution nevertheless exists, albeit with $\lambda$ complex.

- if $d>d_{c}$, there is no real solution either.

4.3. Supercritical case $(\mu>1)$. We must slightly modify the asymptotic behavior at infinity in this case by substracting a non-zero asymptotic limit corresponding to the zero of the potential term. Defining accordingly $\bar{\rho}=((\mu-1) / C)^{1 /(\gamma-1)}$, let $p(x)-\bar{\rho}=\widetilde{p}(x)$. Then $\widetilde{p}(x)$ solves $\frac{1}{2} \widetilde{p}^{\prime \prime}+\frac{d-1}{2 x}-h(\widetilde{p}+\bar{\rho})=0$ which for small $\tilde{p}$ (large $x)$ is $\frac{1}{2} \widetilde{p}^{\prime \prime}+\frac{d-1}{2 x}-h^{\prime}(\bar{\rho}) \widetilde{p}=0$, recalling $h(\bar{\rho})=0$. Recall $h^{\prime}(\bar{\rho})=(\mu-1)(\gamma-1)>0$.

The large $x$ (small $\widetilde{p}$ ) solutions of $\widetilde{p}$ are thus governed by

$$
\frac{1}{2} \widetilde{p}^{\prime \prime}+\frac{d-1}{2 x} \widetilde{p}^{\prime}-h^{\prime}(\bar{\rho}) \widetilde{p}=0,
$$

which can be mapped into a modified Bessel equation as before, but now with $\beta=i \gamma$ and $\gamma=\sqrt{2 h^{\prime}(\bar{\rho})}$.

Proceeding similarly as in the subcritical case, we now get $(\lambda>0)$

$$
\widetilde{p}(x) \sim A_{2} x^{\alpha} K_{\alpha}(\gamma x) \sim \lambda x^{-(d-1) / 2} e^{-\sqrt{2(\mu-1)(\gamma-1)} x} .
$$

Finally, we obtained

Proposition 16. In the supercritical case, $p(x)$ behaves for large $x$ as

$$
p(x) \sim((\mu-1) / C)^{1 /(\gamma-1)}+\lambda x^{-(d-1) / 2} e^{-\sqrt{2(\mu-1)(\gamma-1)} x} .
$$

Again, as compared to the $d=1$ case studied before, the asymptotics of $p(x)$ has an extra $x^{-(d-1) / 2}$ power factor in the corrective term $\widetilde{p}(x)$.

Let us supply a final result pertaining to the supercritical regime: conditionally given the extinction time is finite, the underlying branching process is subcritical with offspring pgf $f_{\rho}(z):=\rho^{-1} f(\rho z)$, obeying $f_{\rho}(1)=1, f_{\rho}^{\prime}(1)=f^{\prime}(\rho)<1$.

Recalling $f(z)=1-\mu(1-z)+C(1-z)^{\gamma}$ where $\mu / \gamma>C>\mu-1$ and $\gamma \in(1,2)$, we indeed get $f_{\rho}^{\prime}(1)=f^{\prime}(\rho)=\mu-C \gamma \bar{\rho}^{\gamma-1}=\mu-\gamma(\mu-1)<1$. Defining $h_{\rho}(z):=$ $f_{\rho}(1-z)-(1-z)$, we get

$$
h_{\rho}(z)=(1-\mu) z+C \frac{\bar{\rho}^{\gamma}}{\rho}\left(\left(1+\frac{\rho}{\bar{\rho}} z\right)^{\gamma}-1\right),
$$

which is regular near $z=1$. 
We have $f_{\rho}(z) \sim_{z=0} \rho^{-1}(1-\mu+C)+(\mu-C \gamma) z+\rho C \gamma(\gamma-1) z^{2} / 2+O\left(z^{3}\right)$, so we are in the domain of attraction of the subcritical model studied in Section 3.1. Defining $\mu_{\rho}:=\mu-\gamma(\mu-1)$, and applying the results of Section 3.1, we conclude that

Proposition 17. Conditionally given that the supercritical branching process survives

$$
p(x) \sim \frac{\lambda}{\bar{\rho}} x^{-(d-1) / 2} e^{-\sqrt{2\left(1-\mu_{\rho}\right)} x}=\frac{\lambda}{\bar{\rho}} x^{-(d-1) / 2} e^{-\sqrt{2(\gamma-1)(\mu-1)} x}, \lambda>0,
$$

displaying the modified scale factor $x_{c}=1 / \sqrt{2(\gamma-1)(\mu-1)}$.

Remark: Let:

$$
x_{V}=\left(\frac{\pi^{d / 2}}{\Gamma(d / 2+1)}\right)^{-1 / d} \text { and } x_{S}=\left(\frac{2 \pi^{d / 2}}{\Gamma(d / 2)}\right)^{-1 /(d-1)} .
$$

If the epidemics starts at distance $x$ of the origin, the tail probability of its spatial extension in $d$-dimensional space for which the volume of a ball is $V=\left(x / x_{V}\right)^{d}$ will be: $\mathbf{P}(V>v) \sim p\left(x_{V} v^{1 / d}\right), v>0$ large, where the large $x$ behaviors of $p(x)$ are given in Propositions $10-12$ and 14. Similarly, the tail probability of the area of the boundary $S$ of the $d$-dimensional sphere $V$ will be $\mathbf{P}(S>s) \sim p\left(x_{S} v^{1 /(d-1)}\right)$, $s>0$ large.

Acknowledgments: The authors thank the referees for their helpful suggestions to improve this presentation. T. H. acknowledges partial support from the labex MME-DII (Modèles Mathématiques et Économiques de la Dynamique, de l' Incertitude et des Interactions).

\section{REFERENCES}

[1] Adke, S. R. The maximum population size in the first $N$ generations of a branching process. Biometrics 20, (1964), 649-651.

[2] Arguin, L-P.; Bovier, A.; Kistler, N. An ergodic theorem for the frontier of branching Brownian motion. Electron. J. Probab. 18, no. 53, 25 pp, (2013).

[3] Bowman, F. Introduction to Bessel functions. Dover Publications Inc., New York, 1958.

[4] Bramson, M. D. Maximal displacement of branching Brownian motion. Comm. Pure Appl. Math. 31, no. 5, 531-581, (1978).

[5] Belavin, A., Polyakov, A.M., Zamolodchikov, A.B.: Infinite conformal symmetry in two dimensional quantum field theory. Nucl. Phys. B241, 33 (1984).

[6] Bender C.M. ; Boettcher S. and Moshe M. Spherically-Symmetric Random Walks in Noninteger Dimension. J. Math. Phys. 35, (1994), 4941-4963.

[7] Comtet, L. Advanced Combinatorics. D. Reidel Publishing Company, Dordrecht, Holland, 1974.

[8] Dawson, D. A.; Iscoe, I.; Perkins, E. A. Super-Brownian motion: path properties and hitting probabilities. Probab. Theory Related Fields 83, no. 1-2, 135-205, (1989).

[9] Dumonteil, E.; Majumdar, S. N.; Rosso, A. and Zoia, A. Spatial extent of an outbreak in animal epidemics. PNAS (2013), vol. 110 no. 11, 4239-4244.

[10] Dynkin, E. B. An introduction to branching measure-valued processes. CRM Monograph Series, 6. American Mathematical Society, Providence, RI. x+134 pp, 1994.

[11] Göing-Jaeschke, A.; Yor, M. A survey and some generalizations of Bessel processes. Bernoulli 9 (2003), no. 2, 313-349.

[12] Graversen, S. E.; Peskir, G. Maximal inequalities for Bessel processes. J. Inequal. Appl. 2 (1998), no. 2, 99-119.

[13] Harris, T. E. The theory of branching processes. Die Grundlehren der Mathematischen Wissenschaften, Bd. 119 Springer-Verlag, Berlin; Prentice-Hall, Inc., Englewood Cliffs, N.J. 1963. 
[14] Inselberg, A. On determinants of Toeplitz-Hessenberg matrices arising in power series. J. Math. Anal. Appl. 63 (1978), no. 2, 347-353.

[15] Kolmogorov, A.; Petrovsky, I. and Piskounov, N. Etude de l'équation de la diffusion avec croissance de la quantité de matière et son application à un problème biologique. Moscou Univ. Bull. Math. 1, pp. 125, (1937).

[16] Koroliouk, V. S. (Ed). Aide-mémoire de théorie des probabilités et de statistique mathématique. V. Koroliouk, N. Portenko, A.Skorokhod... [et al.], Moscou : Editions Mir, 1 vol. (580 p.). Translated from Russian by Djilali Embarek : "Spravocnik po teorii veroâtnostej i matematiceskoj statistike", (1983).

[17] Lamperti, J. An occupation time theorem for a class of stochastic processes. Trans. Amer. Math. Soc. 88, (1958), 380-387.

[18] Revuz , D.; Yor, M. Continuous martingales and Brownian motion, volume 293 of Grundlehren der Mathematischen Wissenschaften [Fundamental Principles of Mathematical Sciences]. Springer-Verlag, Berlin,1991.

[19] Li, Z. Measure-valued branching Markov processes. Probability and its Applications (New York). Springer, Heidelberg. xii+350 pp, 2011.

[20] Sawyer, S.; Fleischman J. Maximum geographic range of a mutant allele considered as a subtype of a Brownian branching random field, PNAS, USA, Vol. 76, no 2, (1979), pp. 872875.

Laboratoire de Physique Théorique et Modélisation, CNRS, UMR-8089 and University of Cergy-Pontoise, 2, rue Adolphe Chauvin F-95302, Cergy-Pontoise, Cedex, FranCe, E-MAIL(S):AVAN@U-CERGY.FR, NICOLAS.GROSJEAN@U-CERGY.FR, HUILLET@U-CERGY.FR 